\title{
Teaching Benign Skin Lesions as a Strategy to Improve the Triage Amalgamated Dermoscopic Algorithm (TADA)
}

\author{
Elizabeth V. Seiverling, MD, Hadjh T. Abrns, MD, Amrit Greene, MD, \\ Melissa Butt, MPH, Oriol Yélamos, MD, Stephen W. Dusza, DrPH, and \\ Ashfaq A. Marghoob, MD
}

Introduction: Dermoscopy aids family physicians (FPs) in skin cancer detection. The triage amalgamated dermoscopic algorithm (TADA) was created to simplify the dermoscopic evaluation of a skin growth. The purpose of this image-based study was to evaluate the effect of teaching the clinical and dermoscopic features of benign skin lesions on the diagnostic accuracy of skin cancer identification using TADA. We also sought to determine the best method to teach benign neoplasms.

Methods: In this cross-sectional study of an educational intervention, FPs participated in dermoscopy training. Participants were divided into 3 groups for teaching of common benign neoplasms (dermatofibroma, angioma, and seborrheic keratosis/lentigo): didactic + interactive, didactic + heuristic, and didactic. For each group, the benign teaching was followed by skin cancer identification training with TADA. All participants took a 30 image pre-test and 30 image post-test.

Results: Fifty-nine participants completed the study. The mean preintervention score (out of 30 correct responses) was $17.9(\mathrm{SD}, 4.5)$ and increased to $23.5(\mathrm{SD}, 3.0)$ on the postintervention evaluation $(P<.001)$. Sensitivity for skin cancer increased from $62.5 \%$ to $88.1 \%$ following the intervention.

Postintervention specificity for skin cancer was $\mathbf{8 7 . 8 \%}$. Sensitivity and specificity increased following the intervention for all 3 types of benign neoplasms. Diagnostic accuracy was not impacted by the method of benign teaching.

Conclusion: Short dermoscopy training sessions with dedicated time for benign growths followed by TADA training for malignant growths are an effective means of teaching FPs dermoscopy and result in a high sensitivity and specificity for the identification of benign and malignant skin neoplasms. ( $\mathrm{J}$ Am Board Fam Med 2019;32:96-102.)

Keywords: Cross-Sectional Studies, Dermatofibroma, Dermoscopy, Family Physicians, Seborrheic Keratosis, Skin Cancer

Current estimates predict that 1 in 5 Americans will develop skin cancer in their lifetime. ${ }^{1}$ Although melanoma is the deadliest form of skin cancer,

This article was externally peer reviewed.

Submitted 9 February 2018; revised 6 August 2018; accepted 10 August 2018.

From Maine Medical Center, Division of Dermatology, Portland, ME (EVS); Department of Family \& Community Medicine (HTA) and Department of Dermatology (AG, MB), Penn State Milton S. Hershey Medical Center, Hershey, PA; Dermatology Department, Hospital Clínic de Barcelona, Universitat de Barcelona, Barcelona, Spain (OY); Dermatology Service, Memorial Sloan Kettering Cancer Center, New York, NY (OY, SWD, AAM).

Funding: Funding for a research assistant and project manager was provided by the James and Joyce Marks Endowment (Penn State Dermatology). when found in its early stages, cure rates reach nearly $100 \% .{ }^{1}$ One of the greatest opportunities for early detection of skin cancer resides within the hands of family physicians (FPs), who see patients that may not otherwise be regularly followed for

Conflict of interest: Dr. Marghoob has served as a consultant for $3 \mathrm{GEN}$, Canfield, and Heine. However, he did not receive support from them for any portion of this research or manuscript. He has received honoraria from $3 \mathrm{GEN}$ and is friends with employees at 3GEN and Canfield. He has intellectual passion for dermoscopy. The other authors have no conflicts of interest to disclose.

Corresponding author: Elizabeth V. Seiverling, MD, Maine Medical Center, Division of Dermatology, Portland, ME 04106 (E-mail: vseiverling@gmail.com). 
total body skin examination by a dermatologist. However, studies have shown that FPs desire better training in dermatology and triaging cutaneous lesions. ${ }^{2,3}$

Dermoscopy is a noninvasive, in vivo, technique that uses a handheld instrument (dermatoscope), which uses polarized light to facilitate visualization of subsurface skin structures (epidermis, dermoepidermal junction, and papillary dermis) that are otherwise not visible. Dermoscopy has vastly improved the diagnostic accuracy of the observer in the detection of cutaneous malignancies. ${ }^{4,5}$

It is, therefore, a valuable tool that can aid FPs in the detection of skin cancer. However, few dermoscopic algorithms were created for this target population of physicians. To address this issue, we previously described a dermoscopic algorithm known as triage amalgamated dermoscopic algorithm (TADA). ${ }^{6}$ TADA requires minimal requisite knowledge of dermoscopy and is designed to facilitate triage of suspect lesions for monitoring, biopsy/ referral, or reassurance. In a pilot, TADA was taught to a group of physicians (64 dermatologists, 19 FPs, and 22 other primary care providers) attending a dermoscopy course and showed good sensitivity and specificity for malignant skin lesions (94.8\% and $72.3 \%$, respectively). ${ }^{7}$ Although no sensitivity differences were observed among specialists, specificity was higher among dermatologists $(79 \%$ vs $72 \%, P=.008)$. In addition, there were no differences in sensitivity or specificity depending on the years of dermoscopy experience. ${ }^{7}$

The difference in specificity may be due to a better knowledge of the clinical features of benign skin growths among dermatologists. Thus, considering the majority of skin lesions that are evaluated in the clinical setting are benign lesions, we hypothesized that teaching the clinical and the dermoscopic features of the commonest benign lesions (dermatofibroma, angioma, and seborrheic keratosis/lentigo) would improve the specificity of the algorithm and, therefore, would improve the diagnostic accuracy of TADA when used by FPs. The objectives of our study were to (1) evaluate the effect of teaching the clinical and dermoscopic features of benign skin lesions on the sensitivity and specificity of TADA; (2) determine which is the best method to teach benign skin lesions: heuristic teaching, didactic teaching, or interactive cases; and (3) confirm the ability of FPs to use TADA to
Figure 1. Study design. Three arms, each with a different educational modality for benign growths, namely dermatofibroma (DF), angioma, and seborrheic keratosis (SK).

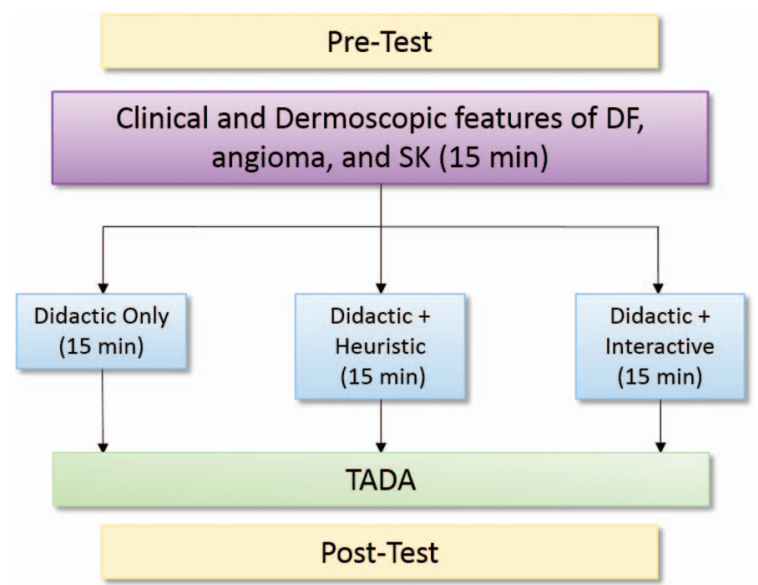

identify dermoscopic images of skin cancer (prior pilot study only included 19 FPs).

\section{Methods}

\section{Study Population and Selection of Skin Lesions}

This study was considered exempt by the Penn State Hershey Institutional Review Board. At Penn State Health, practicing FPs volunteered to participate in dermoscopy training. They were divided into 3 groups based on practice site location (Hershey Medical Center, Mt. Nittany Medical Center, and St. Joseph Medical Center) for teaching of common benign skin growths, namely dermatofibroma, angioma, and seborrheic keratosis/lentigo. The 3 groups (sites) received different teaching interventions for the benign growths: didactic + interactive, didactic + heuristic, and didactic (Hershey Medical Center, Mt. Nittany Medical Center, and St. Joseph Medical Center, respectively). For each group, the benign teaching was followed by skin cancer identification training using TADA. All participants took a 30 question pre-test and 30 question post-test (Figure 1). Images were not repeated, and none of the test images were used in the teaching sessions. The study lesions were selected from dermoscopically imaged neoplasms stored in the Memorial Sloan Kettering Cancer Center imaging database and the dermoscopy teaching database collected by author EVS at Penn State Health. Images were reviewed by EVS, AKG, and HTA, and 60 images were deemed representative exam- 
Figure 2. Common benign skin growths.

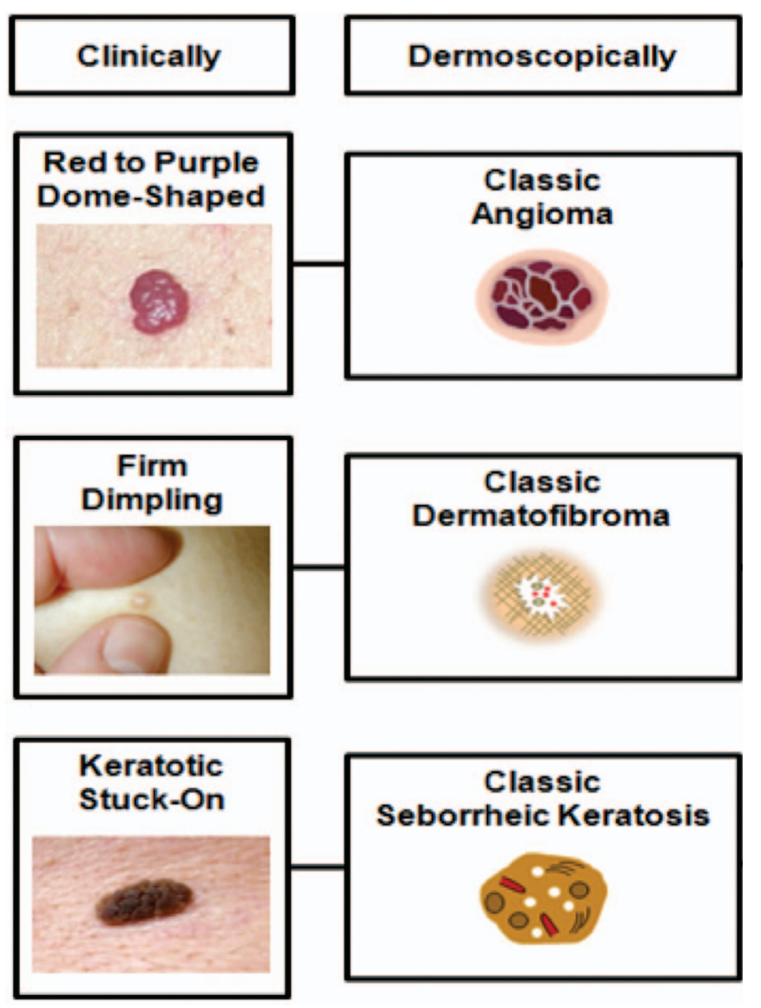

ples of benign and malignant neoplasms and, thus, were selected for inclusion in the pretests and posttests.

The didactic training for the 3 groups was the same and involved reviewing the classic clinical and dermoscopic features of dermatofibroma, angioma, and seborrheic keratosis/lentigo for 15 minutes (Figure 2). Two of the groups then received additional benign neoplasm training. The additional training was either 15 minutes on benign growths by using a heuristic approach or 15 to 30 minutes on benign growths by using an interactive approach. For the heuristic training, images of benign growths were labeled with only the diagnosis and displayed without any explanation of the dermoscopic features. For the interactive arm of the study, participants were asked to describe to the group what they saw, and the salient dermoscopic features of the case were highlighted by the instructor. For all groups, the benign teaching was followed by TADA training on the dermoscopic findings of malignant skin growths (Figure 3). Following the benign and TADA teaching, participants completed a 30 question post-test.
Figure 3. Dermoscopic findings in malignant skin growths by using the Triage Amalgamated Dermoscopic Algorithm TADA.

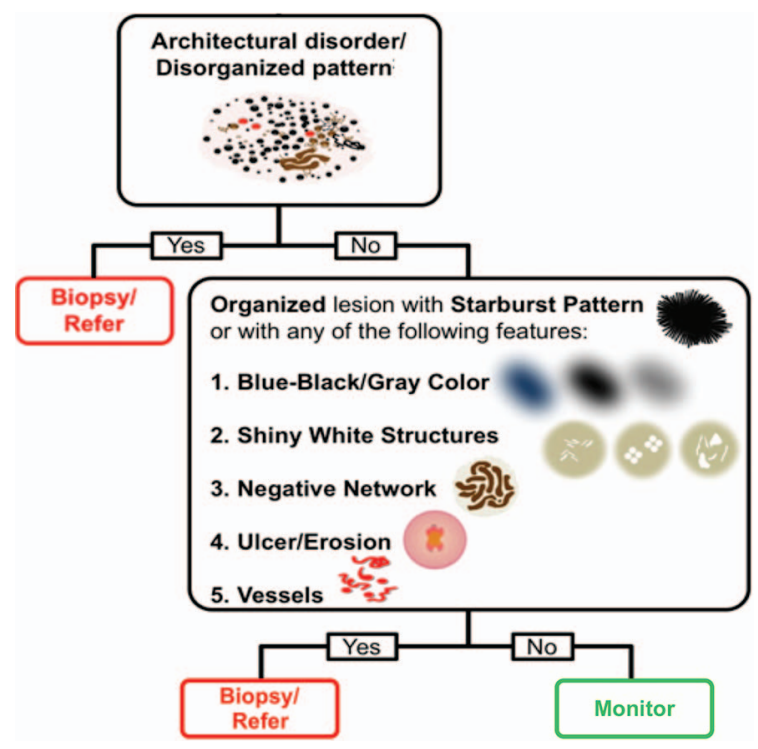

\section{Evaluation Methods}

To evaluate the effectiveness of this intervention, participants were given a pre-test and post-test. The pre-test contained a short demographics section. Participants were then shown 30 dermoscopic images and asked to classify the image as a melanocytic nevus, dermatofibroma, seborrheic keratosis/ lentigo, angioma, or skin cancer. The post-test consisted of a different set of 30 images with the same answer choices. Both the pre-test and the post-test consisted of 16 benign images and 14 malignant images. Correct answers were coded as a 1 and incorrect answers were coded as 0. Evaluation forms were linked together so matched analyses could be performed and analyses were stratified based on intervention type.

\section{Statistics}

Descriptive statistics were used to assess the characteristics of the study population as well as the lesions that were used in the evaluations. For the purposes of this study, certain demographic variables (such as years evaluating skin lesions, prior dermoscopy use or training, and access to a dermatoscope) were assessed dichotomously (Table 1). Evaluation scores were calculated with a total possible score of 30 . Descriptive comparisons of scores were made by using mean scores along with standard deviations and were compared using the Wil- 
Table 1. Population Characteristics

\begin{tabular}{|c|c|c|c|c|}
\hline Variable & Coding & $\begin{array}{c}\text { Hershey Medical Center } \\
\text { (didactic + interactive), } \\
\text { N (\%) }\end{array}$ & $\begin{array}{l}\text { Mt. Nittany Medical } \\
\text { Center (didactic + } \\
\text { heuristic), N (\%) }\end{array}$ & $\begin{array}{c}\text { St. Joseph's Medical } \\
\text { Center (didactic), } \\
\text { N (\%) }\end{array}$ \\
\hline \multicolumn{2}{|l|}{ Number of participants } & $37(100)$ & $15(100)$ & $7(100)$ \\
\hline \multirow[t]{2}{*}{ Sex } & Male & $24(64.9)$ & $8(5.7)$ & $3(42.9)$ \\
\hline & Female & $13(35.1)$ & $7(5.2)$ & $4(57.1)$ \\
\hline \multirow[t]{7}{*}{ Age } & $\leq 20$ & $0(0)$ & $0(0)$ & $0(0)$ \\
\hline & 21 to 30 & $4(10.8)$ & $0(0)$ & $0(0)$ \\
\hline & 31 to 40 & $13(35.1)$ & $3(21.4)$ & $2(28.6)$ \\
\hline & 41 to 50 & $7(18.9)$ & $4(28.6)$ & $2(28.6)$ \\
\hline & 51 to 60 & $6(16.2)$ & $4(28.6)$ & $1(14.3)$ \\
\hline & 61 to 70 & $7(18.9)$ & $3(21.4)$ & $2(28.6)$ \\
\hline & $71+$ & $0(0)$ & $0(0)$ & $0(0)$ \\
\hline \multirow[t]{3}{*}{ Practitioner type } & Physician assistant & $2(5.4)$ & $2(13.3)$ & $0(0)$ \\
\hline & Nurse practitioner & $2(5.4)$ & $4(26.7)$ & $2(28.6)$ \\
\hline & MD/DO & $33(89.2)$ & $9(60.0)$ & $5(71.4)$ \\
\hline \multirow{2}{*}{$\begin{array}{l}\text { Any formal training in } \\
\text { dermoscopy? }\end{array}$} & Yes & $11(29.7)$ & $5(33.3)$ & $0(0)$ \\
\hline & No & $26(70.3)$ & $10(66.7)$ & $7(100.0)$ \\
\hline \multirow{2}{*}{$\begin{array}{l}\text { Do you have access to a } \\
\text { dermatoscope? }\end{array}$} & Yes & $29(78.4)$ & $15(100.0)$ & $5(71.4)$ \\
\hline & No & $8(21.6)$ & $0(0)$ & $2(28.6)$ \\
\hline \multirow{2}{*}{$\begin{array}{l}\text { How many years have } \\
\text { you been evaluating } \\
\text { skin lesions? }\end{array}$} & $\leq 10$ & $18(48.7)$ & $6(40.0)$ & $1(14.3)$ \\
\hline & $>10$ & $19(51.3)$ & $9(60.0)$ & $9(60.0)$ \\
\hline
\end{tabular}

MD, medical doctor; DO, doctor of osteopathic medicine.

coxon-signed rank test. Random effects regression was used to assess differences in preintervention and postintervention scores, controlling for previous dermoscopy training, access to a dermatoscope, sex, participant institution, and years evaluating skin lesions. Sensitivity (the true positive rate) and specificity (the true negative rate) were calculated to measure diagnostic accuracy along with the $95 \%$ confidence intervals (CIs). Separate preintervention and postintervention sensitivities and specificities were calculated for all diagnoses. All analyses were performed using Stata v. 14.0, StataCorp, College Station, TX.

\section{Results}

In total, 59 participants completed both preintervention and postintervention evaluations. The majority of participants were male $(59.3 \%, \mathrm{n}=35)$, did not report any formal dermoscopy training (72.9\%), but did report having access to a dermatoscope $(83.0 \%)$. The median number of years evaluating skin lesions was 15 . Overall, the mean preintervention score (out of 30 correct responses) was $17.9(\mathrm{SD}, 4.5)$ and increased to $23.5(\mathrm{SD}, 3.0)$ on the postintervention evaluation $(P<.001)$ (Fig- ure 4). Almost all participants increased their evaluation score between preintervention and postintervention time points. A significant increase of 5.6 points (95\% CI, 4.6 to 6.4; $P<.001$ ) was observed between study time points. Having some prior formal dermoscopy training was associated with a slightly higher score postintervention $(2.0 ; 95 \% \mathrm{CI}$, $0.14-3.92 ; P=.04)$. No significant differences were observed by the other potential study covari-

\section{Figure 4. Boxplot of preintervention and postintervention scores.}

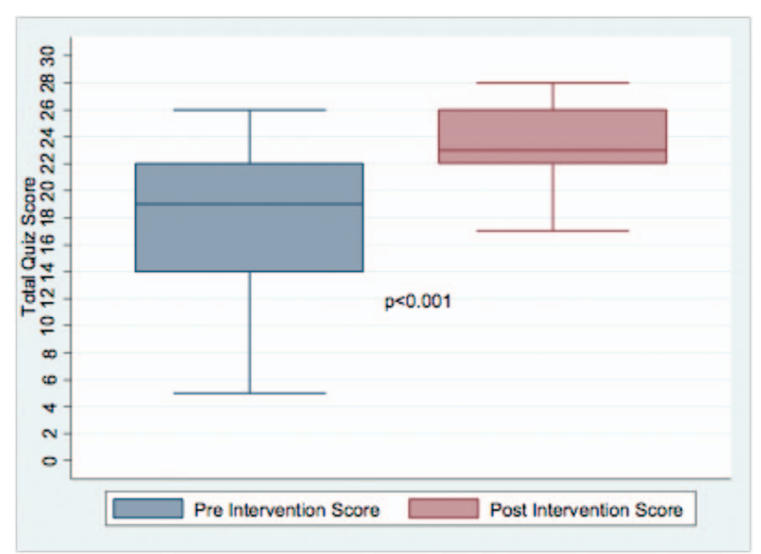


Figure 5. Sensitivity preintervention and postintervention for common benign skin growths and skin cancer. DF, dermatofibroma; SK, seborrheic keratosis.

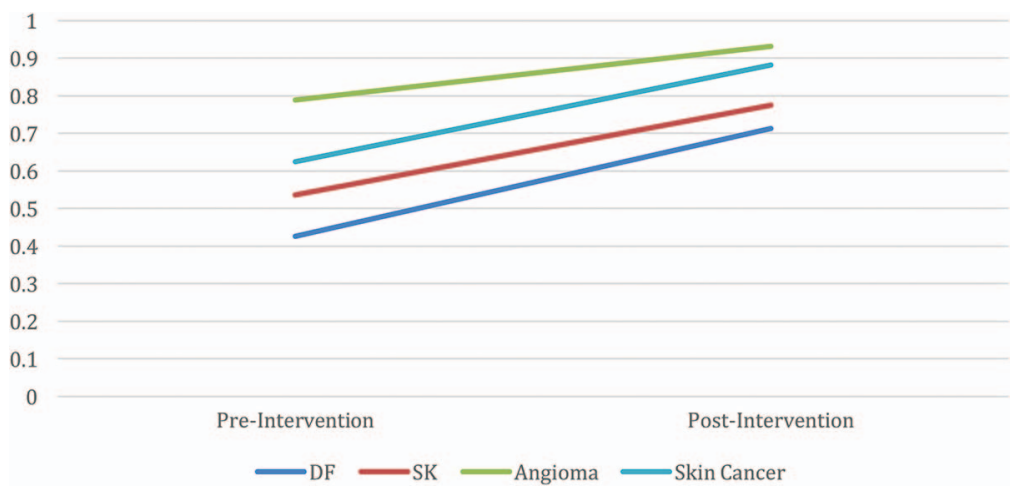

ates (participant sex, age group, access to a dermatoscope, and years evaluating skin lesions), with all observed effects being small and all estimated $P$ values being $>.05$. There was no statistically significant difference in postintervention scores between the 3 groups despite each group undergoing a different teaching modality for the benign portion of the training.

Before the teaching intervention, sensitivity for skin cancer image identification was $62.5 \%$ (95\% CI, $59.1 \%-65.8 \%$ ). Following the intervention (benign and TADA training), the sensitivity for skin cancer image identification increased to $88.1 \%$ (95\% CI, $85.7 \%-90.3 \%$ ) (Figure 5). Postintervention specificity for skin cancer image identification was $87.8 \%$ (95\% CI, 85.6\%-89.8\%); this was a nonsignificant decrease from the pre-test specificity of $90.3 \%$. Sensitivity and specificity increased following the intervention for the identification of all 3 types of benign neoplasms, namely dermatofibroma, angioma, and seborrheic keratosis (Figure 5). Before the intervention, the specificity for dermatofibroma was $88.8 \%$, angioma was $95.2 \%$, and seborrheic keratosis was $87.4 \%$. Following the intervention, the specificity improved, with dermatofibroma at $96.2 \%$, angioma at $97.7 \%$, and seborrheic keratosis at $90.2 \%$.

\section{Discussion}

Up to $25 \%$ of primary care visits are due to a dermatologic condition. ${ }^{8}$ A significant portion of these visits is for a concerning skin growth. ${ }^{9}$ However, FPs are not always confident in their skin cancer detection skills. ${ }^{10}$ Furthermore, over half of FPs report a desire for additional training to aid in skin cancer detection. ${ }^{11}$ Dermoscopy can assist FPs in accurately detecting melanoma. ${ }^{12}$ Because of its proven utility, dermoscopy has been proposed to be added to the FP residency curriculum. ${ }^{13}$ However, the best modality to teach dermoscopy to FPs is not known. Much of the published literature on dermoscopy training is intended for dermatologists and requires prior knowledge of dermoscopic structures. Many FPs are novice dermoscopists and lack the necessary dermoscopy background to use complex algorithms. ${ }^{14}$ Thus, dermoscopy training for FPs should likely differ from dermoscopy training for dermatologists. There have been attempts to simplify dermoscopy algorithms to make them easier to use by novice dermoscopists. Most of these algorithms were designed for melanoma detection and failed to address the dermoscopic findings of basal cell carcinoma and squamous cell carcinoma (which are far more common than melanoma) or did not account for amelanotic melanomas. ${ }^{15-17}$ To address the shortcomings of prior algorithms and to simplify triage, TADA was created and previously described in this journal. ${ }^{7}$ The original TADA pilot had participants from many different specialties and only a small number of FPs $(\mathrm{N}=19)$. The study we present here was conducted with only FPs $(\mathrm{N}=59)$. Our findings confirm that TADA is an effective means of teaching FPs to identify dermoscopic images of skin cancer. Sensitivity for skin cancer identification before the training was $62.5 \%$ and increased to $88.1 \%$ following benign and TADA teaching. Following the training, participants also had very high specificity for malignant growths, $87.8 \%$. This specificity is much higher than the pilot TADA study (72\%). The improved specificity for skin cancer identification in our study may be due to the dedicated benign training we gave before TADA teaching. This training included clinical and dermoscopic images of dermatofibroma, angioma, and seborrheic 
keratosis/lentigo (Figure 2). Clinical images were not part of the pilot study. We suspect that the dedicated benign teaching with clinical images allowed participants to filter out benign growths and, thus, not subject them to further evaluation. Given the high negative predictive value following the intervention for all 3 types of benign growths (dermatofibroma, 97.9\%; angioma, 98.9\%; and seborrheic keratosis, $96.3 \%$ ), benign training might result in the removal of fewer benign growths in clinical practice.

We also sought to determine if there was a difference in outcomes based on the teaching style by which the participants were taught about benign neoplasms. We found no significant difference between the 3 groups, didactic + interactive, didactic + heuristic, and didactic. This finding is consistent with a prior study that found no difference in teaching dermoscopy to novices with an analytic versus heuristic approach. ${ }^{18}$

Lastly, our participants were actively practicing FPs. We wanted to make their participation meaningful but not lengthy. Our training was 75 minutes in total and still achieved powerful changes in diagnostic accuracy. Thus, our study supports prior reports showing basic dermoscopy training of short duration is effective in teaching dermoscopy novices how to triage skin growths. ${ }^{19}$

Furthermore, dermoscopy training is only useful if one has access to a dermatoscope and knows how to operate the device. Thus, at each of our teaching interventions, we conducted 10 minutes of hands-on training on how to operate a dermatoscope. We highlighted how to toggle between polarized and nonpolarized light (there are structures in TADA that can only be seen when using polarized light). We also worked with the medical directors at Penn State Health clinic sites to facilitate the purchase of a dermatoscope for the participants' offices. Lastly, through our work with FPs, we learned that some FPs use an otoscope to look at skin growths instead of a dermatoscope. These tools are not equivalent. Otoscopes do not allow for the visualization of subsurface structures, which are important for skin cancer detection.

\section{Conclusion}

TADA is a valuable triage algorithm for the identification of dermoscopic images of skin cancer. Teaching benign neoplasms may improve malignant neoplasm identification with TADA. Dermos- copy training of common benign skin growths, TADA for skin cancer detection, and training on how to operate a dermatoscope can all be conducted in just over 1 hour. Future dermoscopy courses should include dedicated time addressing the features of benign neoplasms. Furthermore, our study found no difference in the teaching modalities for benign neoplasms; thus, the course director can choose which modality they prefer.

This study confirms FPs can reliably differentiate dermoscopic images of dermatofibroma, angioma, and seborrheic keratoses from malignant neoplasms after TADA training. This training method could be implemented at more FP training sites. Furthermore, dermoscopy training with TADA has the potential to reduce the number of biopsies being performed to detect skin cancer, thus improving patient care and reducing health care costs. Lastly, by equipping FPs with improved knowledge of benign and malignant neoplasms, there might be fewer referrals to dermatology, allowing for faster access to specialty care. Future studies are needed to analyze the effect of this type of dermoscopy training in a clinical setting.

\section{Limitations}

This study was conducted in a classroom setting. It is not known how the participants will apply the training in a clinical setting. The images were of high quality and evaluated on a screen, making it easier to see dermoscopic findings compared with when dermoscopy is performed in vivo. Another limitation to our study is the lack of a dedicated control group. We compared our results to a prior TADA pilot study during which participants received different training, and thus, the improvement we noted in specificity might not be due to only the additional dedicated benign training.

We wish to thank Colin Muraika and Sarah Erlikh for their hard work on data entry.

To see this article online, please go to: bttp://jabfm.org/content/ 32/1/96.full.

\section{References}

1. Society AC. Cancer Facts \& Figures 2017. Atlanta (GA): American Cancer Society; 2017.

2. Robinson S, Kljakovic M, Barry P. Choosing to biopsy or refer suspicious melanocytic lesions in general practice. BMC Fam Pract 2012;13:78. 
3. Kerr O, Walker J, Boohan M. General practitioners opinions regarding the need for training in dermatology at undergraduate and postgraduate levels. Clin Exp Dermatol 2006;31:132-3.

4. Vestergaard M, Macaskill P, Holt P, Menzies S. Dermoscopy compared with naked eye examination for the diagnosis of primary melanoma: a meta-analysis of studies performed in a clinical setting. $\mathrm{Br} \mathrm{J}$ Dermatol 2008;159:669-76.

5. Wozniak-Rito A, Zalaudek I, Rudnicka L. Dermoscopy of basal cell carcinoma. Clin Exp Dermatol 2018;43:241-7.

6. Jaimes N, Marghoob A. Dermoscopic algorithms for skin cancer triage. Available from: https://www. uptodate.com/contents/dermoscopic-algorithmsfor-skin-cancer-triage? source $=$ search_result \& search $=$ dermoscopy\&selectedTitle $=5 \sim 55$. Published 2017.

7. Rogers T, Marino M, Dusza S, et al. A clinical aid for detecting skin cancer: the triage amalgamated dermoscopic algorithm (TADA). J Am Board Fam Med 2016;29:694-701.

8. Perera E, Xu C, Manoharan S. Real-life teledermatology cases. In: Soyer H, Binder M, Smith A, Wurm $\mathrm{E}$, eds. Telemedicine in dermatology. Berlin: Springer Science \& Business Media; 2011. P. 123-9.

9. Kerr O, Tidman M, Walker J, Aldridge R, Benton E. The profile of dermatological problems in primary care. Clin Exp Dermatol 2010;35:380-3.

10. Kirsner R, Muhkerjee S, Federman D. Skin cancer screening in primary care: Prevalence and barriers. J Am Acad Dermatology 1999;41:564-6.

11. van Rijsingen $M$, van Bon B, van der Wilt G, LagroJanssen A, Gerritsen M. The current and future role of general practitioners in skin cancer care: an as- sessment of 268 general practitioners. Br J Dermatol 2014;170:1366-8.

12. Westerhoff K, McCarthy W, Menzies S. Increase in the sensitivity for melanoma diagnosis by primary care physicians using skin surface microscopy. Br J Dermatol 2000;143:1016-20.

13. American Academy of Family Physicians. Recommended Curriculum Guidelines for Fam Med Residents: Conditions of the Skin. Leawood, (KS): American Academy of Family Physicians; 2017.

14. Morris J, Alfonso S, Hernandez N, Fernandez M. Use of and intentions to use dermoscopy among physicians in the United States. Dermatol Pract 2017;7:7-16.

15. Soyer H, Argenziano G, Zalaudek I, et al. Threepoint checklist of dermoscopy: A new screening method for early detection of melanoma. Dermatology 2004;208:27-31.

16. Luttrell M, Hofmann-Wellenhof R, Fink-Puches R, Soyer H. The AC rule for melanoma: a simpler tool for the wider community. J Am Acad Dermatology 2011;65:1233-4.

17. Rosendahl C, Cameron A, McColl I, Wilkinson D. Dermatoscopy in routine practice- "chaos and clues.” Aust Fam Physician 2012;41:482-7.

18. Tschandl P, Kittler H, Schmid K, Zalaudej I, Argenziano G. Teaching dermatoscopy of pigmented skin tumours to novices: comparison of analytic vs. heuristic approach. J Eur Acad Dermatol Venereol 2015; 29:1198-204.

19. Chevolet I, Hoorens I, Janssens A, et al. A short dermoscopy training increases diagnostic performance in both inexperienced and experienced dermatologists. Australas J Dermatol 2015;56:52-5. 